'Laboratorio de Hemodinamia. Unidad de Cardiología. Hospital San Juan de Dios. Santiago, Chile. ${ }^{2}$ Departamento de Medicina, Universidad de Chile. Santiago, Chile.

${ }^{3}$ Escuela de Salud Pública, Universidad de Chile. Santiago, Chile.

${ }^{4}$ Departamento de Enfermedades Respiratorias. Hospital San Juan de Dios. Santiago, Chile. ${ }^{5}$ Servicio de Kinesiología, Centro Diagnóstico y Terapéutico. Hospital San Juan de Dios. Santiago, Chile.

Trabajo no recibió financiamiento.

Los autores declaran no tener conflictos de interés.

Recibido el 19 de marzo de 2018 , aceptado el 18 de diciembre de

Correspondencia a:

Dr. Pablo Sepúlveda Varela Centro de Terapia Endovascular Pontificia Universidad Católica de Chile Marcoleta 367 piso 2 Santiago 8330024 Chile. pablosep@gmail.com

\section{Angioplastía con balón para el tratamiento de la hipertensión arterial pulmonar por enfermedad tromboembólica crónica. Resultados hemodinámicos y clínicos inmediatos}

\author{
PABLO SEPÚLVEDA ${ }^{1,2}$, JUAN ORTEGA ${ }^{1}$, GERMAN ARMIJO ${ }^{1}$, \\ JOSÉ TORRES ${ }^{1}$, PABLO RAMÍREZ ${ }^{1}$, CHRISTIAN BACKHOUSE ${ }^{1}$, \\ CÉSAR VARGAS ${ }^{1}$, LEONEL LÓPEZ ${ }^{1,2}$, FELIPE GONZÁLEZ1, \\ ÁNGEL PUENTES ${ }^{1,2}$, HERNÁN DONOSO ${ }^{1,2}$, AUGUSTO BELLET ${ }^{1,2}$, \\ DIEGO GODOY ${ }^{1}$, MARIO ARAYA ${ }^{1}$, CARMEN LUZ ANDRADE ${ }^{2,4}$, \\ JUAN PABLO MOLINA ${ }^{5}$, CAROLINA NAZZAL ${ }^{3}$
}

\section{Balloon pulmonary angioplasty for the treatment of chronic thromboembolic pulmonary hypertension}

Background: Balloon pulmonary angioplasty (BPA) is a therapeutic alternative for patients with inoperable chronic thromboembolic pulmonary hypertension (CTEPH). Aim: To report the initial experience with the "refined BPA technique" with the use of intravascular images. Patients and Methods: Between June 2015 and June 2016 we selected fourteen patients with CTEPH who were considered candidates for BPA. Lesions targeted for treatment were further analyzed using intravascular imaging with optical frequency domain imaging (OFDI). We report the immediate hemodynamic results and four weeks of follow-up of the first eight patients of this series. Results: We performed 16 BPA in eight patients aged $61 \pm 14$ years ( $88 \%$ women). Mean pulmonary artery pressure (PAPm) was $48.6 \pm 5.8 \mathrm{mmHg}$. Success was achieved in seven patients (88\%). A mean of 2.3 segments per patient were intervened in 11 sessions (1.6 sessions/ patient). Only one patient developed lung reperfusion injury. No mortality was associated with the procedure. After the last BPA session, PAPm decreased to $37.4 \pm 8.6 \mathrm{mmHg}(p=0.02)$. Pulmonary vascular resistance $(R V P)$ decreased from 858,6 $\pm 377,0$ at baseline to $516,6 \pm 323,3$ Dynes $/ \mathrm{sec} \mathrm{cm}^{-5}(p<0.01)$ and the cardiac index increased from $2.4 \pm 0.6$ at baseline to $2.8 \pm 0.3 \mathrm{~L} / \mathrm{min} / \mathrm{m}^{2}(p=0.01)$. At 4 weeks after the last $B P A, W H O$ functional class improved from $3.3 \pm 0.5$ to $2.5 \pm 0.5(p<0,01)$ and six minutes walking distance from $331 \pm 92$ to $451 \pm 149$ $m(p=0.01)$. Conclusions: BPA guided by OFDI for the treatment of inoperable $C T E P H$ patients is a safe alternative with excellent immediate hemodynamic and clinical results.

(Rev Med Chile 2019; 147: 426-436)

Key words: Angioplasty; Hypertension, Pulmonary; Pulmonary Embolism. 


\section{I}

a Hipertensión Pulmonar por Enfermedad Tromboembólica Crónica (CTEPH) es una $\checkmark$ causa rara y potencialmente curable de hipertensión arterial pulmonar (HAP) ${ }^{1,2}$. Los mecanismos etiopatogénicos involucran la transformación fibrótica de trombos residuales posterior a un tromboembolismo pulmonar agudo (TEP), que desencadenan fenómenos de remodelado arterial, aumento de la resistencia vascular pulmonar, hipertensión pulmonar y falla ventricular derecha $a^{3-6}$. Su incidencia varía entre 0,9 a $9,1 \%$ a 2 años luego de un TEP, con sobrevida a 3 años de $50 \% \%^{7-9}$. Para el diagnóstico del CTEPH se debe tener un alto índice de sospecha ya que el cuadro clínico es inespecífico ${ }^{7}$. El estudio de tamizaje debe realizarse en aquellos pacientes que posterior a un episodio de TEP, persisten sintomáticos tras tres meses de anticoagulación efectiva. Para ello, la primera línea lo constituye el cintigrama ventilación perfusión (cintigrama V/Q) dado su alta sensibilidad (96$97 \%)$ y especificidad $(90-95 \%)^{10-14}$. Éste puede complementarse con Tomografía Axial Computada de los vasos pulmonares (AngioTAC pulmonar) y/o Angiografía Pulmonar, ésta última considerada el Gold Standard para el diagnóstico, especialmente de la enfermedad de pequeño vaso ${ }^{15,16}$.

El tratamiento de elección del CTEPH es la endarterectomía pulmonar (EAP), cuyos resultados dan cuenta de sobrevida cercana al $90 \%$ a 3 años ${ }^{17-22}$. Para aquellos pacientes que son excluidos de la cirugía por alta comorbilidad o por presencia de lesiones muy distales -aproximadamente $35 \%$ - o para aquellos casos en que la $\mathrm{HAP}$ recidiva luego de la EAP -aproximadamente 25\% - existen dos alternativas terapéuticas: la terapia médica específica para HAP y la Angioplastía Pulmonar con Balón (BPA) ${ }^{23}$.

La BPA es una técnica mínimamente invasiva que posibilita la intervención de segmentos más distales de la circulación pulmonar, muchas veces inalcanzables por EAP. Fue descrita inicialmente por Voorburg en 1988; posteriormente Feinstein y cols. en 2001 publican la primera serie de 18 pacientes, con un alto número de complicaciones, siendo la principal la injuria pulmonar por reperfusión ${ }^{24-26}$. Recientemente, grupos japoneses, han reportado excelentes resultados al modificar la técnica clásica mediante la incorporación de imágenes intravasculares y/o realizando evaluación hemodinámica de las lesiones con el uso de guía de presión ${ }^{27-35}$. Gracias a estas "técnicas refinadas" se han podido seleccionar mejor los segmentos a intervenir, observándose una disminución de las complicaciones, con excelentes resultados en el mediano a largo plazo ${ }^{36,37}$.

En Chile a la fecha no se han publicado series de pacientes tratados con BPA para el manejo del CTEPH. El siguiente trabajo tiene por objetivo reportar la experiencia inicial de nuestro centro con la "técnica refinada" de BPA mediante el uso de imágenes intravasculares obtenidas con tomografía de coherencia óptica por medio de Optical Frequency Domain Imaging (OFDI) al momento de la intervención. Se presentan los resultados hemodinámicos inmediatos y clínicos a las 4 semanas post BPA.

\section{Pacientes y Método}

Entre junio de 2015 y junio de 2016 se controlaron en policlínico de HAP del Hospital San Juan de Dios 25 pacientes con diagnóstico de CTEPH, todos ellos excluidos de EAP tras evaluación por equipo multidisciplinario. Luego de optimizar el tratamiento médico, que incluía asegurar la mayor dosis tolerable de sildenafil, se actualizó el estudio diagnóstico mediante cateterismo cardiaco derecho y angiografía pulmonar (angiógrafo Artis Zee, Siemens $\left.{ }^{\circledR}\right)$. El sondeo cardiaco derecho debía confirmar la presencia de HAP según la definición hemodinámica actual de CTEPH: PAPm $>25 \mathrm{mmHg}$ RVP $>240$ Dynas $/ \mathrm{seg} / \mathrm{cm}^{-5}$ (o $>3$ Unidades Woods [UW]) y presión capilar pulmonar $(\mathrm{PCP})<15 \mathrm{mmHg}^{7}$. En caso de no poder obtener PCP, se consideró la presión de fin de diástole del ventrículo izquierdo, con el mismo valor de referencia. La preselección del candidato a BPA se basó en la presencia de lesiones sugerentes de CTEPH en la angiografía pulmonar, que incluían: i) redes o hendiduras intravasculares; ii) disminución abrupta del calibre del vaso; iii) suboclusión; iv) oclusión a nivel segmentario o subsegmentario de la circulación pulmonar ${ }^{25,26,38}$. Preferimos la intervención de lesiones tipo i y ii por sobre las de tipo iii, excluyendo las lesiones tipo iv. Se obtuvo consentimiento informado respetando las normas éticas de la declaración de Helsinki del 2013 y posteriormente se programó la primera sesión de BPA. Se indicó la suspensión de la terapia anticoagulante oral 48 horas antes del procedimiento. 
La BPA se realizó con anestesia local (lidocaina $2 \%, 5$ a $10 \mathrm{cc})$. El acceso vascular fue por vía venosa central (VVC) mediante la instalación de un introductor $\left(8 \mathrm{~F}\right.$, Arrow $\left.^{\circledR}\right)$ con técnica standard. Se utilizó VVC femoral derecha para acceder al pulmón izquierdo y VVC yugular derecha para acceder al pulmón derecho. El acceso por VVC braquial se reservó en caso de no obtener acceso a través de los otros sitios. Luego de la instalación de la VVC, se administró heparina no fraccionada $2.000 \mathrm{UI}$ endovenoso (EV), con refuerzos posteriores de 1.000 UI EV cada hora. Se avanzó un catéter Swan-Ganz (7F, $110 \mathrm{~cm}$, Edwards Lifesciences $\left.{ }^{\circledR}\right)$ para la medición de presiones regionales pre y post procedimiento, y cálculo de gasto cardíaco (GC) por método de termodilución. Sobre una guía Hi-Torque Steelcore $\left(0.018 ”, 300 \mathrm{~cm}\right.$, Abbott $\left.^{\circledR}\right)$ se instaló un introductor Destination $(7 \mathrm{~F}, 90 \mathrm{~cm}$, Terumo ${ }^{\circledR}$ ) el que se ubicó en el tronco de la arteria pulmonar $\mathrm{o}$ alguna de sus ramas principales, según el segmento a intervenir. A través de éste, se avanzó un catéter guía MP1 $\left(6 \mathrm{~F}, 100 \mathrm{~cm}\right.$, Terumo $\left.{ }^{\circledR}\right)$ o JR-4 (6F, $100 \mathrm{~cm}$, Medtronic $\left.{ }^{\circledR}\right)$ el que se posicionó selectivamente en el segmento elegido para la BPA. Luego, se avanzó distalmente por el segmento seleccionado una guía floppy, Runthrough NS Hypercoat $\left(0,014\right.$ ", $180 \mathrm{~cm}$, Terumo $\left.{ }^{\circledR}\right)$ y sobre ésta se avanzó la sonda de OFDI Fastview para adquisición de imágenes en consola Lunawave $\left(\right.$ Terumo $^{\circledR}$ ) con el fin de certificar la presencia de redes o hendiduras intravasculares, principal objetivo a tratar (Figura 1). Posteriormente, se avanzó un balón de angioplastía de acuerdo al diámetro arterial estimado por OFDI, tratando de obtener una relación balón/arteria cercana a 0,8:1,0. Para la angioplastía se utilizaron balones semidistensibles entre 1,5-4,0 mm, inflados a presión nominal. Se procuró no intervenir más de 2 segmentos en la primera sesión de BPA y no más de 3 en las sesiones posteriores. Se consideró exitosa la BPA si se lograba apertura del vaso y/o mejoría del flujo, aunque fuera parcial, asociado a disminución de la PAPm y aumento del retorno venoso en la angiografía. Concluido el procedimiento el paciente se hospitalizó en la Unidad Coronaria para monitorización, siendo dado de alta a las 24 hrs, excepto que existieran complicaciones.

Las siguientes sesiones de BPA se programaron en intervalos de 4-6 semanas, con la intención de lograr PAPm $<30 \mathrm{mmHg}$, considerado el objetivo final. Además de los cambios hemodinámicos inmediatos post-procedimiento, se evaluaron parámetros clínicos que incluyeron: péptico natriurético tipo $\mathrm{B}$ (pro-BNP), test de marcha 6 minutos (TM6M) y clase funcional (CF) de la Organización Mundial de la Salud (OMS) 4 semanas luego de la última sesión de BPA. El tratamiento específico para la HAP se mantuvo sin modificación luego de la intervención.

Por criterios angiográficos, 14 pacientes se preseleccionaron para BPA. Se presentan los resultados inmediatos y el seguimiento a 4 semanas de los primeros 8 pacientes de esta serie, que a la fecha de este reporte habían completado más de una sesión de BPA. El resto de los pacientes se encuentran esperando o han completado recientemente su primera sesión. El análisis de los resultados se realiza en base a la intención de tratar. Para la comparación de los parámetros evaluados se utilizó test de T de Student para muestras no independientes y se consideró un valor $\mathrm{p}<0,05$ como significativo.

Se obtuvo aprobación por parte del Comité de Ética institucional correspondiente.

\section{Resultados}

Se realizaron 16 BPA en 8 pacientes, $88 \%$ mujeres, con edad promedio 61,4 \pm 13,6 años. El tiempo de evolución desde el diagnóstico de CTEPH hasta el ingreso al policlínico de HAP fue de 22,9 $\pm 12,6$ meses. Todos los pacientes se encontraban en CF III o IV de la OMS en su primera evaluación. El TM6M basal fue de 322,5 $\pm 88,2$ metros. En cuanto a los factores de riesgo para CTEPH, el 38\% de los pacientes presentó trombosis venosa profunda y el 75\% TEP. El diagnóstico de CTEPH se sospechó por AngioTAC pulmonar sólo en el 50\% de los casos y por Cintigrafía V/Q en el $88 \%$, siendo confirmado en todos los pacientes mediante angiografía pulmonar. La presión sistólica de la arteria pulmonar (PAPs) estimada por ecocardiografía transtorácica fue de $82,3 \pm 14,4 \mathrm{mmHg}$ y $78,6 \pm 12,8 \mathrm{mmHg}$ por cateterismo cardiaco derecho. La medición invasiva mostró PAPm de 48,6 \pm 5,8 $\mathrm{mmHg}$. Respecto del tratamiento específico para CTEPH, el $100 \%$ de los pacientes se encontraban con terapia anticoagulante oral y sildenafil en dosis promedio de $125 \pm 35 \mathrm{mg} /$ día. Las características clínicas basales de la población estudiada se presentan en la Tabla 1. 


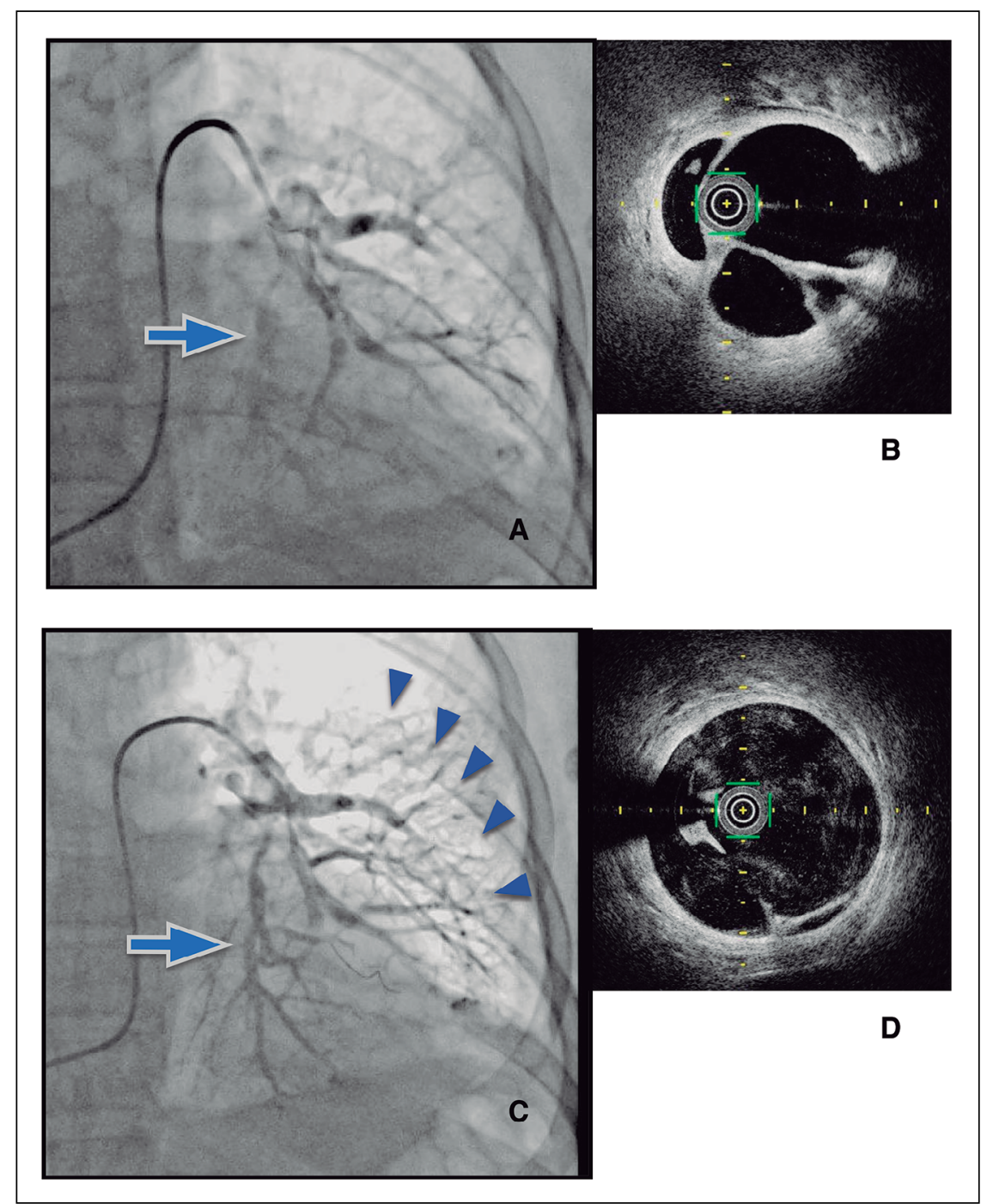

Figura 1. Angiografía y OFDI en BPA. Angiografía pulmonar pre y post BPA $(\mathbf{A}$ y $\mathbf{C})$ y OFDI pre y post BPA (B y D). La flecha identifica uno de los segmentos intervenidos en el lóbulo inferior del pulmón izquierdo. En C, se aprecia la reaparición de flujo y una mayor representación de la circulación pulmonar distal (puntas de flecha), luego de la intervención del segmento adyacente. En $\mathbf{B}$, se aprecian las redes vasculares típicas de las lesiones por CTEPH, que desaparecen luego de la intervención.
La BPA resultó exitosa en 7 pacientes (88\%), logrando en todos ellos una disminución significativa de PAPm. Se consideró en el análisis una paciente que no alcanzó a realizarse la BPA por un hemopericardio ocurrido al ascender los catéteres para la intervención. En los pacientes en que efectivamente se realizó el procedimiento, éste resultó exitoso en el 100\%. En 2 pacientes (29\%) se logró el objetivo final de PAPm $<30 \mathrm{mmHg}$ luego de la primera sesión.

El abordaje para la BPA se realizó por vía femoral en $36,4 \%$, yugular en $45,5 \%$ y braquial en $18,2 \%$. Se intervinieron 2,3 segmentos/paciente (rango 1-4) en 11 sesiones (1,6 sesiones/paciente, rango 1-2). La mayoría de los segmentos intervenidos se localizaron en los lóbulos inferiores y particularmente en el pulmón derecho (Figura 2). Se observaron 2 complicaciones mayores asociadas al procedimiento: un hemopericardio, resuelto con pericardiocentesis; y una perforación de arteria segmentaria post BPA, resuelta por vía endovascular con implante de hidrocoil Azur $\left(\right.$ Terumo $\left.^{\circledR}\right)$. Sólo un paciente presentó injuria de reperfusión, que se resolvió sin necesidad de ventilación mecánica invasiva. No se observó mortalidad asociada al procedimiento, ni en el seguimiento a 30 días. 
Tabla 1. Características clínicas basales

\begin{tabular}{|c|c|c|c|c|c|c|c|c|}
\hline $\begin{array}{l}\text { Paciente/Características } \\
\text { clínicas }\end{array}$ & P1 & $\mathbf{P 2}$ & P3 & P4 & P5 & P6 & P7 & P8 \\
\hline Edad (años) & 44 & 58 & 74 & 74 & 77 & 54 & 43 & 67 \\
\hline Sexo (M/F) & $\mathrm{F}$ & $\mathrm{F}$ & $\mathrm{F}$ & $\mathrm{F}$ & $\mathrm{F}$ & $\mathrm{F}$ & $\mathrm{F}$ & M \\
\hline FRCV & $\begin{array}{l}\text { HTA/DM/ } \\
\text { Dislip }\end{array}$ & No & $\begin{array}{l}\text { HTA } \\
\text { Dislip }\end{array}$ & No & HTA & No & $\begin{array}{l}\text { Obes/ } \\
\text { HTA }\end{array}$ & HTA \\
\hline Otros FR & НіроТ & LES/SAF & No & No & НіроТ & No & No & No \\
\hline Antx TVP (Sí/No) & Sí & No & Sí & No & No & No & Sí & No \\
\hline Antx TEP (Sí/No) & Sí & Sí & No & Sí & Sí & Sí & Sí & No \\
\hline Evolución (meses) & 11 & 30 & 40 & 10 & 24 & 24 & 37 & 7 \\
\hline Clase funcional OMS (1-4) & 4 & 3 & 4 & 3 & 3 & 3 & 4 & 4 \\
\hline TM6M (m) & 356 & 390 & 240 & 333 & 360 & 460 & 240 & 201 \\
\hline BORG post TM6M (0-10) & 8 & 8 & 10 & 8 & 10 & 4 & 10 & 10 \\
\hline pro-BNP (pg/ml) & 9.867 & 160 & 1.916 & 2.242 & 363 & 509 & 111 & 3.000 \\
\hline PSAP eco (mmHg) & 67 & 77 & 77 & 89 & 100 & 98 & 60 & 90 \\
\hline TAPSE (mm) & 14 & 23 & 28 & 15 & 18 & 20 & 24 & 11 \\
\hline Cintigrama V/Q (+/-) & + & + & + & + & + & + & + & - \\
\hline Angio TAC (+/-) & + & + & - & - & + & + & - & - \\
\hline \multicolumn{9}{|l|}{ Tratamiento específico } \\
\hline Riociguat & No & No & No & No & No & No & No & No \\
\hline TACO (Sí/No) & Sí & Sí & Sí & Sí & Sí & Sí & Sí & Sí \\
\hline $02(L / \min ) \times(h)$ & $2 \times 18$ & No & No & No & No & No & No & No \\
\hline Filtro Vena Cava (Sí/No) & No & Sí & No & No & No & No & Sí & No \\
\hline \multicolumn{9}{|l|}{ Tratamiento inespecífico } \\
\hline Furosemida (Sí/No) & No & Sí & Sí & No & No & Sí & No & Sí \\
\hline Espironolactona (Sí/No) & Sí & Sí & Sí & Sí & Sí & Sí & No & Sí \\
\hline Betabloqueantes (Sí/No) & No & No & Sí & No & No & No & No & Sí \\
\hline Digoxina (Sí/No) & Sí & Sí & No & No & No & No & No & No \\
\hline
\end{tabular}

P: Paciente; M/F: masculino, femenino; FR: factores de riesgo; FRCV: factores de riesgo cardiovascular; HTA: hipertensión arterial; Dislip: dislipidemia; Obes: obesidad; LES: lupus eritematoso sistémico; HipoT: hipotiroidismo; SAF: síndrome anti-fosfolípido; TVP: trombosis venosa profunda; TEP: tromboembolismo pulmonar; OMS: Organización Mundial de la Salud; TM6M: Test de marcha de 6 min; BORG: escala de disnea de Borg; pro-BNP: péptido natrurético; PSAP eco: presión sistólica de la arteria pulmonar medida por ecocardiograma transtorácico; TAPSE: excursión del plano del anillo tricúspideo en sístole; Cintigrama V/Q: cintigrama ventilación/perfusión; Angio TAC: angiografía pulmonar por tomografía computada; TACO: tratamiento anticoagulante oral; (+): positivo para CTEPH; (-): negativo para CTEPH.

Comparado con el basal, y luego de la última sesión de BPA, la PAPm disminuyó de 48,6 \pm 5,8 a $37,4 \pm 8,6 \mathrm{mmHg}(\mathrm{p}=0,0149)$, la RVP disminuyó de 858,6 $\pm 377,0$ a 516,6 \pm 323,3 Dynas/seg/ $\mathrm{cm}^{-5}(\mathrm{p}=0,0002)$ y el índice cardíaco aumentó de $2,4 \pm 0,6$ a $2,8 \pm 0,3 \mathrm{~L} / \mathrm{min} / \mathrm{m}^{2}(\mathrm{p}=0,0112)$. Las características hemodinámicas basales y los resultados inmediatos se muestran en la Tabla 2 y Figura 3.

A las 4 semanas posterior a la última sesión de BPA se observó una mejoría de la CF de $3,3 \pm 0,5$ a 2,5 $\pm 0,5(\mathrm{p}=0,0072)$ y del TM6M de $331 \pm 92 \mathrm{a}$ $451 \pm 149 \mathrm{~m}(\mathrm{p}=0,0027)$. EL pro-BNP disminuyó de $2.271 \pm 3.046$ a $1.101 \pm 115 \mathrm{pg} / \mathrm{dl}(\mathrm{p}=0,3571)$. Los resultados clínicos inmediatos se muestran en la Figura 4. 


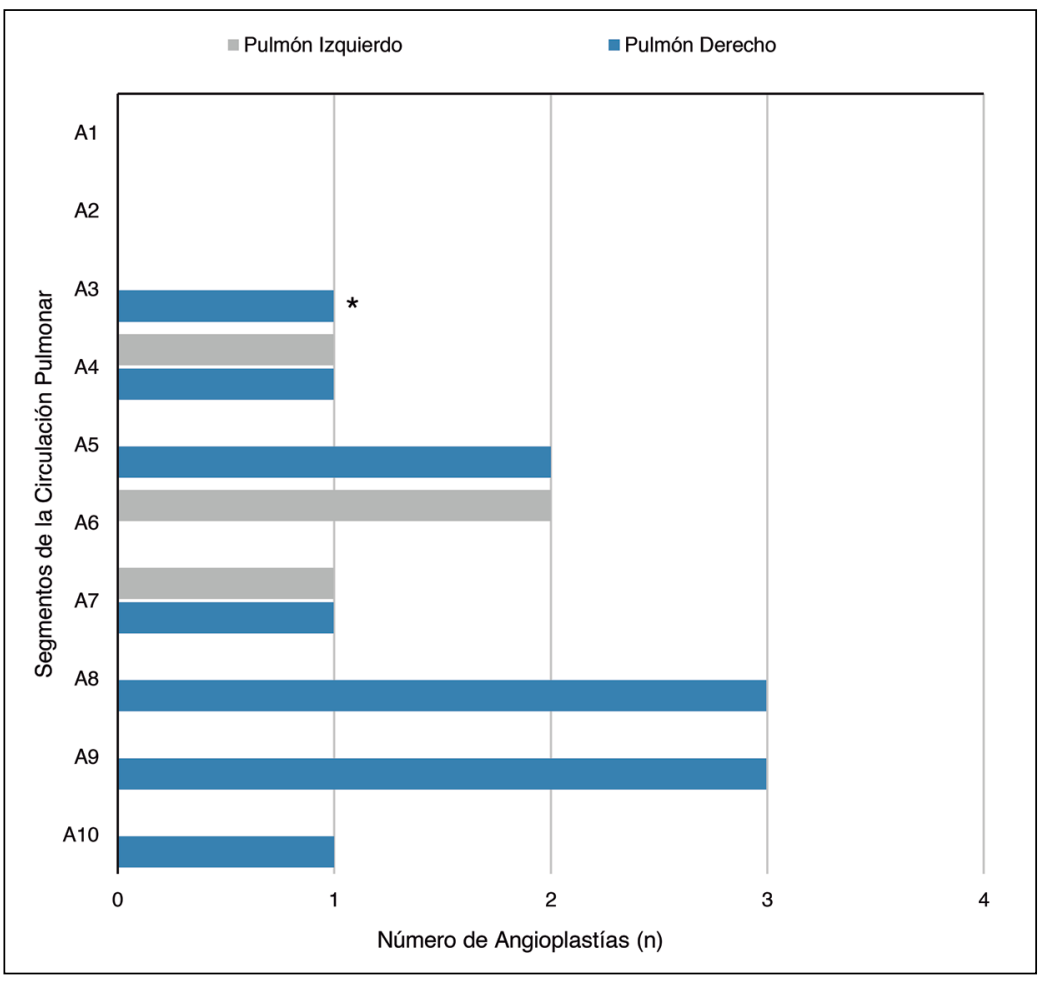

Figura 2. Segmentos pulmonares intervenidos. Distribución de las 16 BPA de la serie, de acuerdo a los segmentos de la circulación pulmonar intervenidos y el número de angioplastías realizadas por segmento. Se aprecia una preferencia por intervención en segmentos basales y del pulmón derecho, debido a la facilidad de acceso y el mayor impacto en la circulación pulmonar. * La única complicación (perforación arterial), se observó al intervenir el segmento $A 3$, en el lóbulo superior del pulmón derecho.

Tabla 2. Características hemodinámicas basales

\begin{tabular}{|c|c|c|c|c|c|c|c|c|c|c|}
\hline Paciente & 1 & 2 & 3 & 4 & 5 & 6 & 7 & 8 & prom & DE \\
\hline FC (Ipm) & 93 & 78 & 68 & 72 & 64 & 70 & 80 & 70 & 75,3 & 9,5 \\
\hline PAs $(\mathrm{mmHg})$ & 133 & 115 & 120 & 120 & 110 & 122 & 180 & 145 & 132,1 & 24,1 \\
\hline PAd $(\mathrm{mmHg})$ & 82 & 85 & 80 & 80 & 70 & 80 & 91 & 77 & 80,7 & 6,5 \\
\hline PAm $(\mathrm{mmHg})$ & 99 & 95 & 93 & 93 & 83 & 94 & 121 & 93 & 96,9 & 11,7 \\
\hline $\mathrm{ADm}(\mathrm{mmHg})$ & 12 & 16 & 6 & 8 & 7 & 9 & 3 & 17 & 10,3 & 5,0 \\
\hline VDs $(\mathrm{mmHg})$ & 62 & 65 & 73 & 67 & 74 & 100 & 91 & 89 & 78,3 & 14,9 \\
\hline VDfd $(\mathrm{mmHg})$ & 15 & 30 & 10 & 10 & 8 & 8 & 10 & 8 & 12,7 & 8,0 \\
\hline PAPs (mmHg) & 64 & 70 & 76 & 64 & 80 & 95 & 86 & 91 & 78,6 & 12,8 \\
\hline PAPd $(\mathrm{mmHg})$ & 27 & 41 & 25 & 29 & 41 & 25 & 21 & 33 & 31,0 & 7,7 \\
\hline PAPm $(\mathrm{mmHg})$ & 39 & 54 & 43 & 43 & 53 & 51 & 47 & 53 & 48,6 & 5,8 \\
\hline $\mathrm{PCP}(\mathrm{mmHg})$ & 15 & 15 & 12 & 4 & 10 & 8 & 10 & 9 & 10,1 & 3,9 \\
\hline GC (L/min) & 5,8 & 5,5 & 4,3 & 3,0 & 3,6 & 4,3 & 4,3 & 3,3 & 4,3 & 1,1 \\
\hline IC $\left(\mathrm{L} / \mathrm{min} / \mathrm{m}^{2}\right)$ & 3,0 & 3,4 & 2,7 & 1,9 & 2,3 & 2,3 & 2,0 & 1,8 & 2,4 & 0,6 \\
\hline RVP (Dynas/seg/cm-5) & 331 & 567 & 576 & 924 & 937 & 1.496 & 688 & 1.067 & 858,6 & 377,0 \\
\hline RVS (Dynas/seg/cm-5) & 1.200 & 1.149 & 1.619 & 2.244 & 1.657 & 1.581 & 2.065 & 1.351 & $1.606,7$ & 420,2 \\
\hline
\end{tabular}

FC: frecuencia cardiaca; PAs: presión arterial sistólica; PAd: presión arterial diastólica; PAm: presión arterial media; ADm: presión media aurícula derecha; VDs: presión sistólica ventrículo derecho; VDfd: presión de fin de diástole ventrículo derecho; PAPs: presión sistólica de arteria pulmonar; PAPd: presión diastólica de arteria pulmonar; PAPm: presión media de arteria pulmonar; PCP: presión capilar pulmonar; GC: gasto cardiaco; IC: índice cardiaco; RVP: resistencia vascular pulmonar; RVS: resistencia vascular sistémica; prom: promedio; DE: desviación estándar. 


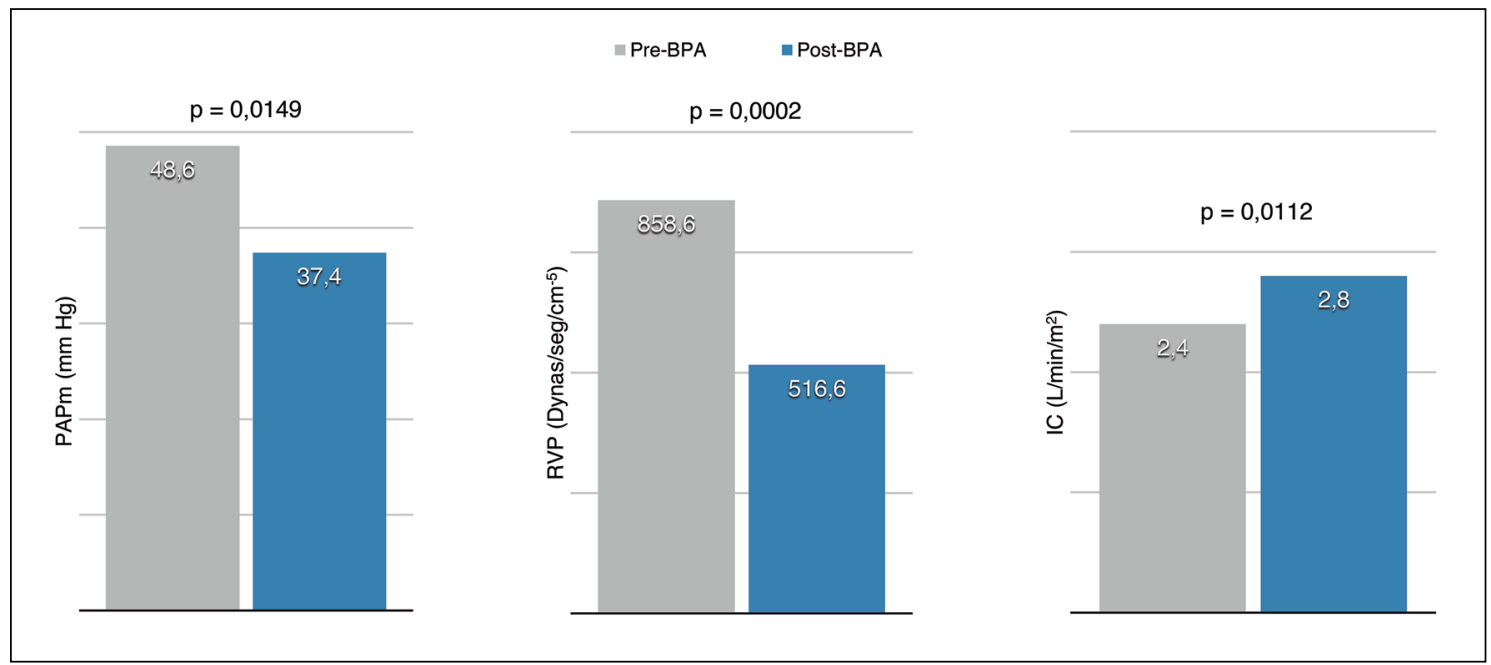

Figura 3. Resultados hemodinámicos.

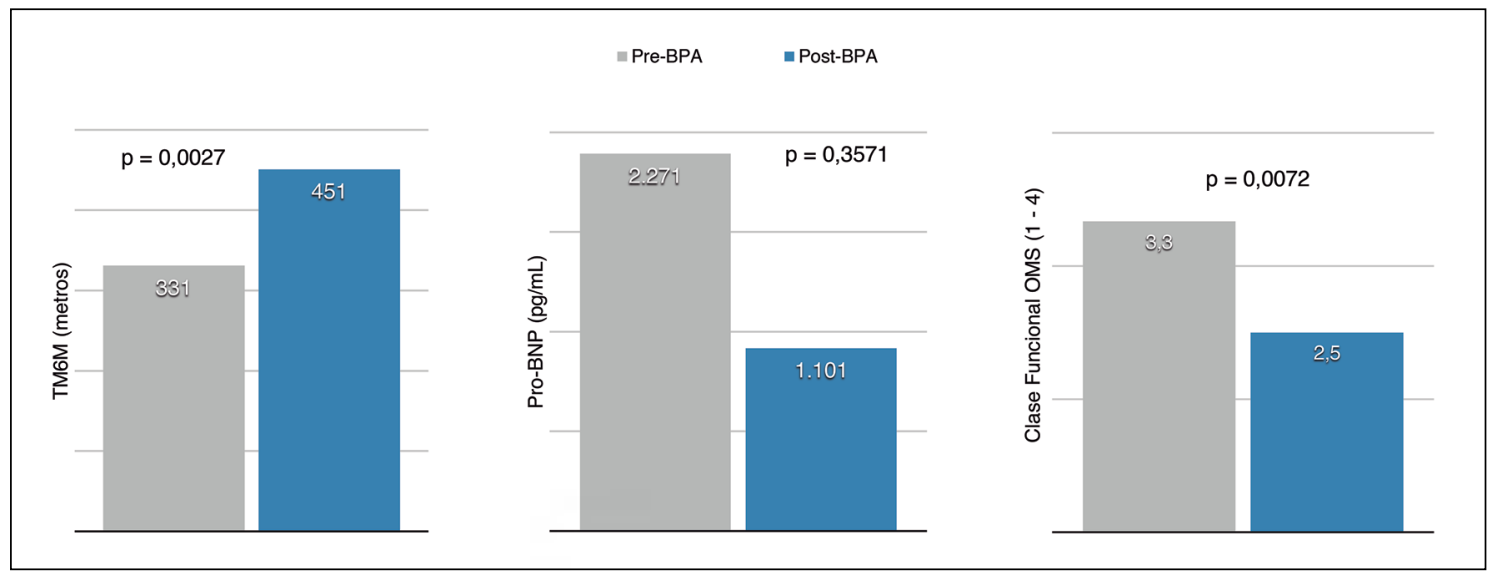

Figura 4. Resultados clínicos.

Tabla 3. Resultados clínicos y hemodinámicos

\begin{tabular}{|lcccc|}
\hline Parámetro & Pre-BPA & Post-BPA & \% Cambio & p \\
\hline PAPm (mmHg) & $48,6 \pm 5,8$ & $37,4 \pm 8,6$ & $-23,0 \%$ & 0,0149 \\
\hline RVP (Dynas/seg/cm $\left.{ }^{-5}\right)$ & $858,6 \pm 377,0$ & $516,6 \pm 323,3$ & $-39,8 \%$ & 0,0002 \\
IC (L/min/m²) & $2,4 \pm 0,6$ & $2,8 \pm 0,3$ & $+14,3 \%$ & 0,0112 \\
Clase funcional OMS (1-4) & $3,3 \pm 0,5$ & $2,5 \pm 0,5$ & $+24,2 \%$ & 0,0072 \\
Pro-BNP (pg/dL) & $2.271 \pm 3.046$ & $1.101 \pm 1.115$ & $-51,5 \%$ & 0,3571 \\
TM6M (m) & $331 \pm 92$ & $451 \pm 149$ & $+26,6 \%$ & 0,0027 \\
\hline
\end{tabular}

Mismas abreviaciones que Tabla 1 y Tabla 2. 
Tabla 4. Comparación otras series iniciales internacionales

\begin{tabular}{|c|c|c|c|c|c|c|c|c|}
\hline \multirow[t]{2}{*}{ Parámetro } & \multicolumn{4}{|c|}{$\begin{array}{c}\text { España }^{39} \text { Año } 2016 \\
\text { N Pac }=7\end{array}$} & \multicolumn{4}{|c|}{ Brasil $^{40} \quad$ Año 2017} \\
\hline & Basal & $\begin{array}{c}\text { N Pac }= \\
\text { Post BPA }\end{array}$ & 7 p & $\begin{array}{c}\% \\
\text { Cambio }\end{array}$ & Basal & $\begin{array}{l}\text { N Pac }=4 \\
\text { Post BPA }\end{array}$ & $\mathbf{p}$ & $\begin{array}{c}\% \\
\text { Cambio }\end{array}$ \\
\hline PAPm $(\mathrm{mmHg})$ & $56 \pm 17$ & $36 \pm 10$ & $<0,6$ & -28 & $41(31-53)$ & $23(17-33)$ & $<0,01$ & -43 \\
\hline RVP (UW) & $11,8 \pm 4$ & $6,1 \pm 2,2$ & $<0,2$ & -41 & $8,21(4,8-14,38)$ & $3,27(2,1-4,8)$ & $<0,01$ & -60 \\
\hline IC $\left(\mathrm{L} / \mathrm{min} / \mathrm{m}^{2}\right)$ & $2,3 \pm 0,4$ & $2,6 \pm 0,6$ & $<0,1$ & 15,7 & $2,31(1,73-2,98)$ & $3,15(2,38-3,44)$ & $<0,01$ & 36 \\
\hline $\begin{array}{l}\text { Clase funcional } \\
\text { OMS (I/II/III/IV) }\end{array}$ & $3,8 \pm 0,2$ & $2,3 \pm 0,2$ & $<0,001$ & 39 & $0 / 0 / 7 / 3$ & $2 / 8 / 0 / 0$ & $<0,05$ & - \\
\hline Pro-BNP (pg/dl) & $1.366 \pm 929$ & $646 \pm 677$ & $<0,1$ & -52 & $472 \pm 357$ & $294 \pm 310,2$ & $<0,03$ & -31 \\
\hline
\end{tabular}

Mismas abreviaciones que Tabla 1 y Tabla 2.

Un resumen de los cambios pre y post BPA en los principales parámetros estudiados, se presenta en la Tabla 3.

\section{Discusión}

Esta es la primera serie nacional publicada de BPA para el tratamiento de CTEPH, en pacientes excluidos de cirugía. Nuestros resultados son similares a lo descrito en otras series iniciales publicadas recientemente (Tabla 4) ${ }^{39,40}$. Todos los pacientes se encontraban en CF III o IV de la OMS, con TM6M disminuido en forma importante y PAPm elevada en grado moderado a severo. Un tercio de los pacientes presentaban disfunción ventricular derecha en la evaluación inicial.

En el diagnostico inicial del CTEPH, el cintigrama V/Q fue el examen de elección, no sólo para la certificación diagnóstica, sino también para planificar la intervención ${ }^{7}$. En esta serie, sólo en un caso el cintigrama V/Q informó alteraciones de la perfusión en segmentos que tanto por angiografía como por OFDI se encontraban sanos. La elección de los segmentos a intervenir se hizo por criterios angiográficos pero que se correlacionaran con segmentos alterados de la perfusión pulmonar. Una vez seleccionados adecuadamente los segmentos a intervenir, el OFDI permitió precisar la existencia de lesiones del tipo "redes" o "hendiduras" y de esta forma, optimizar el procedimiento. Nuestro grupo ha adquirido una importante experiencia en los últimos años en la evaluación de lesiones coronarias con OFDI, por lo que la elección de esta modalidad como parte de la "técnica refinada" nos pareció la más adecuada.

Los resultados en parámetros hemodinámicos y clínicos son consistentes con lo reportado en las principales series internacionales ${ }^{26}$. Los cambios en la sintomatología se aprecian precozmente, como lo revela la mejoría de la capacidad funcional y el incremento de los metros caminados en el TM6M a las cuatro semanas posterior a la última BPA. Sin embargo, a pesar que estos cambios implican una importante mejoría en la calidad de vida, pueden no ser suficientes para pacientes con las severas limitaciones funcionales que impone el CTEPH. Se debe intentar lograr el objetivo hemodinámico final de PAPm $<30 \mathrm{mmHg}$, por las implicancias pronósticas que conlleva. En esta serie, solo un tercio de los pacientes lograron dicho objetivo.

La BPA, resultó ser un procedimiento seguro, sin observarse mortalidad asociada a la intervención y baja tasa de complicaciones. Estas últimas se describen hasta en $7 \%$ y tienen directa relación con el volumen del centro y la experiencia del operador $^{2}$. De particular relevancia es la presencia de un solo caso $(6,25 \%)$ de injuria de reperfusión, que no requirió de ventilación mecánica invasiva y se resolvió exitosamente. El número de sesiones es menor a lo reportado en la literatura (promedio 4-5 sesiones $^{32}$ ) ya que esta serie se presenta con fines comparativos con otras series iniciales $(\mathrm{Ta}-$ bla 4) para evaluar factibilidad, reproducibilidad, seguridad y resultados a corto plazo de la técnica. La totalidad de la serie de pacientes candidatos a BPA, será objeto de un nuevo estudio a futuro y de seguimiento a largo plazo. 
Las principales limitaciones de este trabajo son las propias de una experiencia inicial, no aleatorizada ni ciega, en la que además los pacientes son seleccionados para optimizar el rendimiento de la técnica y disminuir sus complicaciones. A pesar de haber demostrado resultados hemodinámicos significativos en forma inmediata, éstos están lejos de ser definitivos y su impacto real en la población estudiada no podrá apreciarse sino hasta completar todas las sesiones de BPA necesarias para lograr PAPm $<30 \mathrm{mmHg}$. En cuanto al seguimiento, es mandatorio realizar uno a largo plazo con el fin de evaluar el impacto de la técnica en la función ventricular derecha. Si bien el pro-BNP disminuyó posterior a la intervención, no lo hizo en forma significativa, lo que implica indirectamente que los cambios hemodinámicos inmediatos no alcanzan a verse reflejados en la función del ventrículo derecho. Probablemente, mayores sesiones de BPA y un seguimiento más alejado puedan mostrar resultados significativos.

Finalmente, la experiencia aquí presentada resume los resultados de un solo centro, siendo la técnica todavía muy poco difundida en el medio local, por lo que se requiere de validación de estos resultados por otros operadores y/o centros a nivel nacional antes de que ésta pueda ser ofrecida en forma más rutinaria.

En conclusión, la BPA guiada por OFDI, para el tratamiento del CTEPH en pacientes excluidos de EAP, es una alternativa segura y efectiva con excelentes resultados hemodinámicos inmediatos y clínicos a corto plazo.

\section{Referencias}

1. Kim N, Delcroix M, Jenkins D, Channik R, Dartevelle $\mathrm{P}$, Jansa P, et al. Chronic Tromboembolic Pulmonary Hypertension. J Am Coll Cardio 2013; 62 (25 Suppl): D92-9.

2. Piazza G, Goldhaber SZ. Chronic Tromboembolic Pulmonary Hypertension. N Engl J Med 2011; 364: 351-60.

3. Lang IM, Pegamento R, Bonderman D, Yuan JX. Risk factors and basic mechanisms of chronic thromboembolic pulmonary hypertension: a current understanding. Eur Respir J 2013; 41 (2): 462-8.

4. Matthews D, Hemnes A. Current concepts in the pathogenesis of chronic thromboembolic pulmonary hypertension. Pulm Circ 2016; 6 (2): 145-54.

5. Humbert M. Pulmonary arterial hypertension and chronic thromboembolic pulmonary hypertension: pathophysiology. Eur Respir Rev 2010; 19 (115): 59-63.

6. Lang IM, Madani M. Update on Chronic Tromboembolic Pulmonary Hypertension. Circulation 2014; 130 (6): 508-18.

7. Galié N, Humbert M, Vachiery JL, Gibbs S, Lang I, Torbicki A, et al. 2015 ESC/ERS Guidelines for the diagnosis and treatment of pulmonary hypertension: the joint task force for the diagnosis and treatment of pulmonary hypertension of the European Society of Cardiology (ESC) and the European respiratory Society (ERS). Endorsed by: Association for European Paediatric and Congenital Cardiology (AEPC), International Society for Heart and Lung Transplantation (ISHLT). European Heart Journal 2016; 37 (1): 67-119.

8. JCS Joint Working Group. Guidelines for the diagnosis, treatment and prevention of pulmonary tromboembolism and deep vein thrombosis (JCS 2009). Circ J 2011; 75 (5): 1258-81.

9. Lewczuk J, Piszko P, Jagas J, Porada A, Sobkowicz B, Wrabec K, et al. Prognostic factors in medically treated patients with chronic pulmonary embolism. Chest 2001; 119 (3): 818-23.

10. Tanabe N, Sugiura T, Tatsumi K. Recent progress in the diagnosis and management of chronic thromboembolic pulmonary hypertension. Respiratory Investigation 2013. 51 (3): 134-46.

11. Hoeper MM, Barbera JA, Channick RN, Hassoun PM, Lang IM, Manes A, et al. Diagnosis, assessment, and treatment of non-pulmonary arterial hypertension pulmonary hypertension. J Am Coll Cardiol 2009; 54 (Suppl 1): S85-S96.

12. Fedullo PF, Auger WR, Kerr KM, Rubin LJ. Chronic tromboembolic pulmonary hypertension. N Engl J Med 2001; 345: 1465-72.

13. Tunariu N, Gibbs SJ, Win Z, Ging-Sing W, Graham A, Gishen $\mathrm{P}$, et al. Ventilation-perfusion scintigraphy is more sensitive than multidetector CTPA in detecting chronic thromboembolic pulmonary disease as a treatable cause of pulmonary hypertension. J Nucl Med 2007; 48 (5): 680-4.

14. Soler X, Kerr KM, Marsh JJ, Renner JW, Hoh CK, Test VJ, et al. Pilot study comparing SPECT perfusion scintigraphy with CT pulmonary angiography in chronic thromboembolic pulmonary hypertension. Respirology 2012; 17 (1): 180-4.

15. Ryan KL, Fedullo PF, Davis GB, Vasquez TE, Moser KM. Perfusion scan findings understate the severity of angiographic and hemodynamic compromise in chronic thromboembolic pulmonary hypertension. Chest 1988; 93 (6): 1180-5. 
16. Reichelt A, Hoeper MM, Galanski M, Keberle M. Chronic thromboembolic pulmonary hypertension: Evaluation with 64-detector row CT versus digital subtraction angiography. Eur J Radiol 2009; 71: 49-54.

17. Jamieson SW, Kapelanski DP, Sakakibara N, Manecke GR, Thistlethwaite P, Kerr K, et al. Pulmonary endarterectomy: experience and lessons learned in 1,500 cases. Ann Thorac Surg 2003; 76 (5):1457-62.

18. Skoro-Sajer N, Marta G, Gerges C, Hlavin G, Nierlich $\mathrm{P}$, Taghavi S, et al. Surgical specimens, haemodynamics and long-term outcomes after pulmonary endarterectomy. Thorax. 2014; 69 (2): 116-22.

19. Matsuda H, Ogino H, Minatoya K, Sasaki H, Nakanishi $\mathrm{N}$, Kyotani S, et al. Long-term recovery of exercise ability after pulmonary endarterectomy for chronic thromboembolic pulmonary hypertension. Ann Thorac Surg. 2006; 82: 1338-43.

20. Corsico AG, D’Armini AM, Cerveri I, Klersy C, Ansaldo E, Niniano R, et al. Long-term outcome after pulmonary endarterec- tomy. Am J Respir Crit Care Med. 2008; 178 (4): 419-24.

21. Saouti N, Morshuis WJ, Heijmen RH, Snijder RJ. Longterm out- come after pulmonary endarterectomy for chronic thromboembolic pulmonary hypertension: a single institution experience. Eur J Cardiothorac Surg. 2009; 35 (6): 947-52.

22. Madani MM, Auger WR, Pretorius V, Sakakibara N, Kerr KM, Kim NH, et al. Pulmonary endarterectomy: recent changes in a single institution's experience of more than 2,700 patients. Ann Thorac Surg. 2012; 94 (1): 97-103.

23. Ghofrani H, D’Armini A, Grimminger F, Hoeper M, Jansa P, Kim NH, et al. Riociguat for the treatment of chronic thromboembolic pulmonary hypertension. CHEST-1 Study Group. N Engl J Med 2013; 369: 31929.

24. Voorburg JA, Cats VM, Buis B, Brunschke AV. Balloon angioplasty in the treatment of pulmonary hypertension caused by pulmonary embolism. Chest 1988; 94 (6): 1249-53.

25. Feinstein JA, Goldhaber SZ, Lock JE, Fernandes SM, Landzberg MJ. Balloon pulmonary angioplasty for treatment of chronic thromboembolic pulmonary hypertension. Circulation 2001; 103 (1): 10-3.

26. Ogo T. Ballon pulmonary angioplasty for inoperable chronic thromboembolic pulmonary hypertension. Curr Opin Pulm Med 2015; 21 (5): 425-31.

27. Sugimura K, Fukumoto Y, Satoh K, Nochioka K, Miura $\mathrm{Y}$, Aoki T, et al. Percutaneous transluminal pulmonary angioplasty markedly improves pulmonary hemodynamics and long-term prognosis in patients with chronic thromboembolic pulmonary hypertension. Circ J 2012; 76 (2): 485-8.

28. Kataoka M, Inami T, Hayashida K, Shimura N, Ishiguro $\mathrm{H}$, Abe T, et al. Percutaneous transluminal pulmonary angioplasty for the treatment of chronic thromboembolic pulmonary hypertension. Circ Cardiovasc Interv 2012; 5: 756-62.

29. Andreassen AK, Ragnarsson A, Gude E, Geiran O, Andersen R. Balloon pulmonary angioplasty in patients with inoperable chronic thromboembolic pulmonary hypertension. Heart 2013; 99 (19): 1415-20.

30. Fukui S, Ogo T, Morita Y, et al. Right ventricular reverse remodelling after balloon pulmonary angioplasty. Eur Respir J 2014; 43: 1394-402.

31. Inami T, Kataoka M, Ando M, Fukuda K, Yoshino H, Satoh T, et al. A new era of therapeutic strategies for \& chronic thromboembolic pulmonary hypertension by two different interventional therapies; pulmonary endarterectomy and percutaneous transluminal pulmonary angioplasty. PLoS One 2014; 9 (4): e94587.

32. Taniguchi $\mathrm{Y}$, Miyagawa $\mathrm{K}$, Nakayama K, Kinutani H, Shinke T, Okada K, et al. Balloon pulmonary angioplasty: An additional treatment option to improve the prognosis of patients with chronic thromboembolic pulmonary hypertension. EuroIntervention 2014; 10 (4): 518-25.

33. Mizoguchi H, Ogawa A, Munemasa M, Mikouchi H, Ito $\mathrm{H}$, Matsubara H. Refined balloon pulmonary angioplasty for inoperable patients with chronic thromboembolic pulmonary hypertension. Circ Cardiovasc Interv 2012; 5 (6): 748-55.

34. Ikeda N, Kubota S, Okazaki T, Hara H, Hiroi Y. Comparison of intravascular optical frecuency domain imaging versus intravascular ultrasound during balloon pulmonary angioplasty in patients with chronic tromboembolic pulmonary hypertension. Catheterization and Cardiovascular Interventions 2016; 87 (7): E268-74.

35. Inami $\mathrm{T}$, Kataoka $\mathrm{M}$, Shimura $\mathrm{N}$, Ishiguro $\mathrm{H}$, Yanagisawa R, Fukuda $\mathrm{K}$, et al. Pressure-Wire-Guided Percutaneous Transluminal Pulmonary Angioplasty. A Breakthrough in Catheter-Interventional Therapy for Chronic Thromboembolic Pulmonary Hypertension. J Am Coll Cardio 2014; 7 (11): 1297-306.

36. Ogawa A, Satoh T, Fukuda T, Sugimura K, Fukumoto $\mathrm{Y}$, Emoto N, et al. Balloon pulmonary angioplasty for chronic thromboembolic pulmonary hypertension: Results of a Multicenter Registry. Circ Cardiovasc Qual Outcomes 2017; 10: e004029.

37. Roik M, Wretowski D, Rowinski O, Kabyk A, Kostrubiec M, Lichodziejewska B, et al. Refined balloon pulmonary angioplasty in inoperable chronic thromboembolic pul- 
monary hypertension - a multimodality approach to the treated lesion. Int J Cardiol 2014; 177 (3): e139-41.

38. Kawakami T, Ogawa A, Miyaji K, Mizoguchi H, Shimokawanahara H, Naito T, et al. Novel Angiographic Classification of Each Vascular Lesion in Chronic Thromboembolic Pulmonary Hypertension Based on Selective Angiogram and Results of Balloon Pulmonary Angioplasty. Circ Cardiovasc Interv 2016; 9 (10): e003318.

39. Velázquez M, Albarrán A, Alonso S, García J, Cortina
JM, Escribano P, et al. Angioplastia pulmonar con balón en la hipertensión pulmonar tromboembólica crónica no operable. Experiencia inicial en España en una serie de 7 pacientes. Rev Esp Cardiol 2015; 68 (6): 535-7.

40. Souza FS, Melo I, Leite M, Abreu R, Ferreira MG, Ribeiro P. First results of balloon pulmonary angioplasty for inoperable chronic tromboembolic pulmonary hypertension (CTEPH) patients in Brazil. International CTEPH Association Congress June 9-10, 2017, Leuven, Belgium. ICC17-50. 